\begin{tabular}{|l|l|l|l|l|}
\hline KLIO & $\mathbf{9 4}$ & 2012 & 1 & $7-17$ \\
\hline
\end{tabular}

\author{
Rita Gautschy (Basel)
}

\title{
Sonnenfinsternisse und ihre chronologische Bedeutung: Ein neuer Sonnenfinsterniskanon für Altertumswissenschaftler ${ }^{1}$
}

In antiken Quellen sind zahlreiche astronomische Ereignisse überliefert. Schon im 18. Jh. wurde erkannt, daß solche Berichte es ermöglichen, absolute Daten zu gewinnen. So konnten etwa die Daten von ca. 1430 v. Chr. an durch die Erwähnung der Sonnenfinsternis vom 15. Juni $763 \mathrm{v}$. Chr. in der assyrischen Eponymenchronik absolut fixiert werden. Für chronologische Zwecke sind vor allem Sonnen- und Mondfinsternisse, Neumond- bzw. Altmondsichtungen, sowie Beobachtungen der Planeten Merkur, Venus, Mars, Jupiter und Saturn wichtig. Im 19. Jh. war im Bereich der Altertumswissenschaften das Interesse an astronomischen Fragestellungen groß. Ginzel (1899) publizierte für chronologische Zwecke einen Kanon der Mond- und Sonnenfinsternisse zwischen 900 v. Chr. und 600 n. Chr. ${ }^{2}$ Es war jedoch Neugebauer (1929; 1937), der mit seinen Werken schließlich den interessierten Altertumswissenschaftlern jenes Werkzeug in die Hand gab, das bis in die 1980 er Jahre verwendet wurde. ${ }^{3}$ Seither sind zahlreiche Computerprogramme verfügbar geworden, die schnell und scheinbar zuverlässig Mond- und Planetenpositionen sowie Finsternisse berechnen und auch darstellen können. Viele dieser Programme sind aber hauptsächlich auf den heutigen Gebrauch ausgerichtet, und weniger darauf, historische Himmelsereignisse möglichst genau zu berechnen. Eines der großen Probleme in der Berechnung historischer Himmelserscheinungen ist die Verlangsamung der Erdrotation, die zu einer Änderung der Tageslänge führt. ${ }^{4}$ Der im Laufe der Zeit durch die langsamere Erdrotation entstandene Zeitunterschied wird $\Delta \mathrm{T}$ genannt. $\Delta \mathrm{T}$ kann nur nachträglich aus Beobachtungen abgeleitet werden. ${ }^{5}$

Ginzel's Arbeit blieb bis heute wegweisend für alle späteren Werke über Finsternisse, die mit Hauptaugenmerk auf deren chronologischen Zweck erstellt wurden. ${ }^{6}$ Ginzel hat in seinem Kanon Zitate antiker Autoren gesammelt und Identifikationen für die einzel-

1 Diese Arbeit wurde vom Schweizerischen Nationalfonds im Rahmen eines Marie Heim-Vögtlin Stipendiums finanziert. Ich danke Leonhard Burckhardt, Oskar Kaelin und Hans-Peter Mathys, die mit Diskussionen und Kommentaren geholfen haben, das Manuskript zu verbessern.

2 Ginzel (1899).

3 Neugebauer (1929) und Neugebauer (1937).

4 Während der letzten 40 Jahre addierten sich diese kleinen Änderungen der Tageslänge auf 30 Sekunden.

${ }^{5} \Delta \mathrm{T}$ beträgt im Jahr 2000 v. Chr. ca. 12 Stunden und die Unsicherheit von $\Delta \mathrm{T} \pm$ eine Stunde.

${ }^{6}$ Der bislang letzte Finsterniskanon, der speziell auf die Bedürfnisse von Altertumswissenschaftlern ausgerichtet ist, wurde von de Meis (2002) publiziert. Dieser Kanon enthält Berechnungen und Karten der Sichtbarkeitsgebiete für Sonnen- und Mondfinsternisse, die in Quellentexten überliefert sind, beginnend 763 v. Chr. und endend $1740 \mathrm{n}$. Chr. Die überlieferten babylonischen Sonnen- und Mondfinsternisbeobachtungen bzw. -berechnungen wurden von Huber/de Meis (2004) zusammen gestellt. Diese beiden Werke enthalten ausschließlich Karten von Finsternissen, die schriftlich belegt sind. 
nen überlieferten Finsternisse vorgeschlagen. Sein Werk enthält Karten für die Gebiete des klassischen Altertums, in denen für jedes Jahrhundert zwischen $900 \mathrm{v}$. Chr. und $600 \mathrm{n}$. Chr. die Totalitätszonen von Sonnenfinsternissen eingezeichnet sind. Allerdings wußte Ginzel noch nichts von der Verlangsamung der Erdrotation; er mußte in seine Berechnungen „empirische Korrekturen“ einfügen, um die Lage der Totalitätszonen von Sonnenfinsternissen in Übereinstimmung mit sicher datierbaren Berichten zu bringen.

\section{Neue Berechnungen}

Der hier vorgestellte elektronische Kanon der Sonnenfinsternisse lehnt sich sehr stark an Ginzel (1899) an. Er enthält Karten aller Sonnenfinsternisse, die im Zeitraum zwischen $2500 \mathrm{v}$. Chr. und $1000 \mathrm{n}$. Chr. im ausgewählten geographischen Bereich zwischen $\left(20^{\circ} \mathrm{N}\right.$, $\left.5^{\circ} \mathrm{O}\right),\left(20^{\circ} \mathrm{N}, 50^{\circ} \mathrm{O}\right),\left(50^{\circ} \mathrm{N}, 5^{\circ} \mathrm{O}\right)$ und $\left(50^{\circ} \mathrm{N}, 50^{\circ} \mathrm{O}\right)$ potentiell auffällig waren und somit für Identifikationen von Finsternisberichten aus diesem geographischen Bereich in Frage kommen, auch wenn sie nicht vorhergesagt wurden. Für die Berechnung der Mondund Sonnenpositionen im Zeitraum zwischen 2500 v. Chr. und $1000 \mathrm{n}$. Chr. wurden die neuesten Langzeit-Ephemeriden des Jet Propulsion Laboratory gewählt, welche es erlauben, die Positionen der Sonne, des Mondes und aller Planeten zwischen 3001 v. Chr. und 3000 n. Chr. zu berechnen. ${ }^{7}$ Für $\Delta \mathrm{T}$ wurden die polynomialen Ausdrücke von Espenak (2004) verwendet, für die Abschätzung der Unsicherheit dieser Werte die Formel von Huber (2006). ${ }^{8}$

Jede Sonnenfinsternis wurde mit drei verschiedenen $\Delta \mathrm{T}$-Werten berechnet, damit die Unsicherheit dieses Wertes in den Ergebnissen berücksichtigt werden kann: einmal mit dem mittleren $\Delta \mathrm{T}$-Wert, der sich aus den Formeln von Espenak (2004) errechnen läßt, einmal mit dem unteren $\Delta \mathrm{T}$-Wert, der dem mittleren $\Delta \mathrm{T}$ minus der Unsicherheit des $\Delta \mathrm{T}$ Wertes entspricht, und einmal mit dem oberen $\Delta \mathrm{T}$-Wert, der dem mittleren $\Delta \mathrm{T}$ plus der Unsicherheit des $\Delta \mathrm{T}$-Wertes entspricht. Für die Orte Athen, Rom, Babylon, Memphis, Theben, Alexandria und Knossos wurden alle diejenigen Sonnenfinsternisse ausgewählt, die bei mindestens einem der drei $\Delta \mathrm{T}$-Werte eine Magnitude von größer oder gleich 0.5 erreichten. ${ }^{9}$ Für jeden dieser Orte wurden die Zeitpunkte des Beginns, des Maximums und des Endes der Sonnenfinsternis, die Magnitude, die Sonnenaufgangs- und Sonnenuntergangszeit, sowie die Positionswinkel und Sonnenhöhen berechnet. Eine Grenzgröße von 0.5 wurde basierend auf den Angaben in Ginzel (1899) gewählt, der schreibt, daß eine unvorhergesagte Sonnenfinsternis erst dann bemerkt wird, wenn eine Magnitude von etwa 0.75 erreicht wird falls die Sonne hoch am Himmel steht, bzw. wenn eine Magnitude von 0.5 erreicht wird falls die Sonne knapp über dem Horizont steht. ${ }^{10}$ Genaue Finsternisvorhersagen sind schriftlich erstmals ab etwa 300 v. Chr. in Babylon greifbar. Ab dann kann man Berichte finden, in denen auch Sonnenfinsternisse mit geringeren Magnituden als 0.5 überliefert sind. Deswegen werden im Kanon in einer separaten Tabelle die Daten derjenigen Sonnenfinsternisse aufgeführt, die im betrachteten geographi-

7 DE-406 Langzeit-Ephemeriden des Jet Propulsion Laboratory, Standish (1998).

8 Espenak (2004) und Huber (2006).

${ }^{9}$ Die Größe oder Magnitude einer Finsternis ist bei einer partiellen Finsternis der Anteil des vom Mond bedeckten Sonnendurchmessers. Bei einer totalen oder ringförmigen Finsternis ist die Magnitude das Verhältnis zwischen Mond- und Sonnendurchmesser. Der Wert ist bei einer totalen Finsternis etwas größer als 1, bei einer ringförmigen knapp kleiner als 1.

10 Ginzel (1899) 14. 
schen Bereich zwar eine geringere Magnitude als 0.5 erreichten, aber dennoch schriftlich belegt sind.

Die Daten des Sonnenfinsterniskanons sind nach Jahrhunderten unterteilt in Tabellen angelegt. Ist eine Sonnenfinsternis schriftlich belegt, so folgt die Quellenangabe, die direkt anklickbar ist und das Zitat sofort im Original (soweit möglich) und in englischer Übersetzung anzeigt. Für jede Sonnenfinsternis ist eine Karte für den geographischen Bereich zwischen $\left(20^{\circ} \mathrm{N}, 5^{\circ} \mathrm{O}\right),\left(20^{\circ} \mathrm{N}, 50^{\circ} \mathrm{O}\right),\left(50^{\circ} \mathrm{N}, 5^{\circ} \mathrm{O}\right)$ und $\left(50^{\circ} \mathrm{N}, 50^{\circ} \mathrm{O}\right)$ verfügbar. In den Karten sind zahlreiche während dieser Periode wichtige Orte eingezeichnet, deren Namen und Koordinaten sich in einem Ortsregister finden lassen. 10 in der entsprechenden Periode wichtige Orte sind jeweils speziell markiert, mit Nummern versehen und rechts außerhalb der Karte benannt. Ist eine Finsternis schriftlich belegt, so gibt es weitere Detailplots für die Gebiete Italien, Griechenland und Kleinasien, Ägypten und Vorderasien. Die feine Unterteilung des Gebiets in $1^{\circ}$-Schritten soll es ermöglichen, auf einfache Weise die Lage eines Ortes zu eruieren, der nicht im Ortsregister eingetragen ist. Die mit Nummern versehenen Orte variieren je nach Periode und ihre Namen und Koordinaten können im Ortsregister nachgeschlagen werden. Der Sonnenfinsterniskanon ist online verfügbar unter:

http://www.gautschy.ch/ rita/archast/solec/finsternis.html (deutsche Version)

http://www.gautschy.ch/ rita/archast/solec/solareclipse.html (englische Version)

Über die Homepage des Seminars für Alte Geschichte in Basel:

http://altegeschichte.unibas.ch/20143

\section{Anwendungsbeispiele}

\section{Die so genannte Ugarit-Sonnenfinsternis}

Auf einer Tontafel, die man im Schutt des Palasttores von Ugarit gefunden hat, befindet sich eine der ältesten Beschreibungen einer Sonnenfinsternis. ${ }^{11}$ Die Lesung des Textes ist nicht unproblematisch, es gibt verschiedene Interpretationen. ${ }^{12}$ Der Text spricht von einem Eintreten der Sonne in ein Tor, was nach mesopotamischer Vorstellung einem normalen Auf- oder Untergang der Sonne entspricht. Das Akkadische kennt einen eigenen Terminus zur Beschreibung von Sonnen- oder Mondfinsternissen, aus dem Ugaritischen haben wir dafür bisher keine Belege. Auf rein philologischer Basis läßt sich eine Textinterpretation als Beschreibung einer Sonnenfinsternis daher nicht rechtfertigen. Entscheidend für eine solche Auffassung ist der kurze Text auf der Rückseite der Tontafel: unter der Annahme, daß der Text auf der Rückseite etwas mit demjenigen auf der Vorderseite zu tun hat, schließt die Erwähnung der beiden Leberschauen eine Beschreibung eines gewöhnlichen Sonnenuntergangs aus. Denn Leberschauen wurden nur bei außergewöhnlichen Ereignissen durchgeführt, nicht bei alltäglichen.

11 KTU 1.78 im Musée National de Damas, Inventarnummer 3689 (Dietrich/Loretz [1976]).

12 Es gibt Autoren, die bezweifeln, daß es sich dabei um eine Beschreibung einer Sonnenfinsternis handelt (Pardee [2000] 416-427; Hunger/Pingree [1999] 11). 
Die folgende Transkription und Übersetzung des Textes stammt von Dietrich \& Loretz: ${ }^{13}$

\author{
Obv. 1: $\quad$ b T'T.ym. HdT \\ Obv. 2: $\quad$ xyr. arbt \\ Obv. 3: $\quad$ SpS. Tg̈rh \\ Obv. 4: $\quad$ rSp \\ Rev. 5: $\quad$ kbdm. tborn \\ Rev. 6: skn \\ (Obv.) Im sechsten (Abschnitt) des Neumondtages des Hiyyār ist unter- \\ gegangen die Sonne, ihr Torhüter ist Rešeph. \\ (Rev.) Zwei Lebern hat man untersucht: Gefahr!
}

Viel diskutiert ist die korrekte Bedeutung vor allem der ersten Zeile. Die Verbindung zwischen der Präposition „b“ und dem Zahlwort „TT“ läßt vermuten, daß es sich beim Zahlwort um eine Ordinalzahl handelt und die erste Zeile somit „am 6. Neumondtag“ übersetzt werden sollte. $\mathrm{Da}$ in ugaritischen Texten jedoch üblicherweise die Ordinalzahl in der Form „TdT“ überliefert wird und zudem auf den ersten Blick diese Übersetzung aus logischen Gründen unmöglich scheint - Neumond ist nur an einem Tag pro Mondmonat -, wurde sie stets verworfen. ${ }^{14}$ Stattdessen schlugen de Jong \& van Soldt (1987-1988) vor, daß es sich beim Zahlwort „TT“ in dieser Konstruktion um ein Zahladjektiv handelt, das sich auf einen Tagesabschnitt bezieht. ${ }^{15}$ Über die Tagesunterteilung in Ugarit weiß man allerdings nichts Genaues. Geht man von ungleichen Tagesstunden aus wie es in Ägypten üblich war, müßte die Sonnenfinsternis in diesem Fall um Mittag herum stattgefunden haben. ${ }^{16}$ Ein Hinweis, daß die Sonne mitten am Tag untergegangen war, läßt wiederum auf eine bedeutende Sonnenfinsternis mit totaler bzw. nahezu totaler Bedeckung schließen.

Bei der Analyse dieses Textes ist jedoch ein Punkt sehr wichtig, den schon Dietrich \& Loretz (2002) genannt haben: ${ }^{17}$ dieser Bericht wurde offensichtlich in Eile niedergeschrieben. Die Unregelmäßigkeit der Buchstaben ist auffällig und doppelte Einstiche eines Keils sind mehrfach feststellbar. Bei KTU 1.78 handelt es sich um ein Palimpsest, solche Dokumente stammen in der Regel aus den Priesterbibliotheken der Akropolis. ${ }^{18}$ Auch die verwendeten Buchstabenformen erinnern an die Dokumente aus der Priesterbibliothek. Bei einem in Eile geschriebenen Text darf eine stichwortartige, abgekürzte Schreibweise und eine wenig ausgefeilte Grammatik erwartet werden. Zudem macht die naheliegende Deutung von „TT“ als Ordinalzahl und die Angabe „am 6. Neumondtag“ durchaus Sinn, wenn man sie nicht auf den Mondmonat, sondern auf die nächst größere Zeiteinheit - das Mondjahr - bezieht: mit dem 6. Neumondtag könnte hier der 6. Monats-

13 Dietrich/Loretz (2002) 64.

14 Dietrich/Loretz (2002) 64-65.

15 De Jong/van Soldt (1987-1988) 68.

16 De Jong/van Soldt (1987-1988) 73-74 legen dar, daß die Angabe, daß in Ugarit eine Sonnenfinsternis am Neumondtag stattfand, die Verwendung eines Kalenders impliziert, der von seiner Natur her dem ägyptischen Kalender ähnlich ist und nicht dem babylonischen. Da der ägyptische Einfluß in Ugarit im 2. Jt. v. Chr. groß war, ist dies nicht überraschend.

17 Dietrich/Loretz (2002) 68-69.

18 Dietrich/Loretz (2002) 69. Sowohl auf der Vorder- als auch auf der Rückseite sind Zeichenreste eines Vortextes erkennbar. 
beginn im Jahr gemeint sein. Die Nennung des Hiyyār an dieser Stelle würde demnach das Ergebnis von De Jong \& van Soldt (1987-1988) stützen, die abgeleitet haben, daß der Hiyyār der sechste Monat im ugaritischen Jahr war. ${ }^{19}$ „Hiyyār“ am Beginn der zweiten Zeile müßte dann als Apposition verstanden werden und eine ausformulierte Übersetzung folgendermaßen lauten:

(Obv.) Am 6. Neumondtag, [dem] des Hiyyār, ist untergegangen die Sonne, ihr Hüter des Tores ist Rešeph.

Mit Rešeph ist vermutlich Mars gemeint. ${ }^{20}$ Das bedeutet, daß Mars während der maximalen Bedeckungsphase über dem Horizont gestanden haben sollte. ${ }^{21}$ Die Monatsangabe im Text, Hiyyār, weist auf eine Finsternis im Februar bzw. März hin. ${ }^{22}$ Da über Schaltungen in Ugarit nichts bekannt ist, diese aber unabdingbar waren, um den belegten Mondkalender in Übereinstimmung mit dem Sonnenlauf zu halten, müssen mögliche Abweichungen vom Februar/März in Betracht gezogen werden. Ich habe die Daten der Neumonde und der letzten und ersten Sichtbarkeiten der Mondsichel vor bzw. nach Neumond für Ugarit berechnet. In der letzten Spalte der folgenden Tabelle 1 sind die ugaritischen Monate aufgelistet, in die die jeweilige Sonnenfinsternis fiel. ${ }^{23}$ Zieht man die Unsicherheit von $\Delta \mathrm{T}$ in Betracht, so können die in der Tabelle 1 aufgelisteten Sonnenfinsternisse innerhalb eines sehr großzügig gewählten historisch möglichen Bereichs eine totale bzw. nahezu totale Bedeckung der Sonne in Ugarit verursacht haben: ${ }^{24}$

\begin{tabular}{|r|c|c|c|c|c|c|r|}
\hline Datum & Typ & Magnitude & Maximum & SA & SU & Mars & Monat \\
\hline 3.5 .1375 & $\mathrm{~T}$ & $0.94-1.01$ & $6: 05$ & $5: 25$ & $18: 36$ & $-5^{\circ}$ & $9 / 8$ \\
\hline 15.7 .1360 & $\mathrm{R}$ & $0.85-0.96$ & $15: 37$ & $4: 46$ & $19: 17$ & $+40^{\circ}$ & $12 / 11 / 10$ \\
\hline 8.1 .1340 & $\mathrm{~T}$ & $0.93-0.97$ & $11: 22$ & $7: 09$ & $16: 53$ & $+28^{\circ}$ & $5 / 4$ \\
\hline 13.3 .1335 & $\mathrm{R}$ & $0.88-0.95$ & $13: 29$ & $6: 21$ & $17: 42$ & $+10^{\circ}$ & $7 / 6$ \\
\hline 27.9 .1261 & $\mathrm{R}$ & $0.90-0.96$ & $16: 05$ & $5: 50$ & $18: 13$ & $-37^{\circ}$ & $2 / 1 / 12$ \\
\hline 5.3 .1223 & $\mathrm{~T}$ & $0.89-1.00$ & $13: 53$ & $6: 28$ & $17: 34$ & $+41^{\circ}$ & $7 / 6$ \\
\hline 21.1 .1192 & $\mathrm{R}$ & $0.88-0.97$ & $13: 37$ & $7: 04$ & $16: 58$ & $-31^{\circ}$ & $6 / 5 / 4$ \\
\hline
\end{tabular}

Tab. 1: Datum (v. Chr.), Typ (T: total; R: ringförmig), Magnitudenbereich, Zeitpunkt der maximalen Bedeckung für mittleres $\Delta \mathrm{T}$, Sonnenaufgangs- (SA) und Sonnenuntergangszeit (SU) berechnet für Ugarit $\left(\lambda=35^{\circ} 47^{\circ} \mathrm{O}, \varphi=35^{\circ} 37^{\prime} \mathrm{N}\right)$ mit einem mittleren $\Delta \mathrm{T}$-Wert, sowie die Höhe des Planeten Mars über dem Horizont zum Zeitpunkt der maximalen Bedeckung und der ugaritische Monat. Die angegebenen Zeiten sind stark abhängig vom $\Delta \mathrm{T}$-Wert.

19 De Jong/van Soldt (1987-1988) 69-71. Das ugaritische Jahr begann in etwa mit der Herbsttagundnachtgleiche, der Monat Hiyyār war der sechste des Jahres.

20 De Jong/van Soldt (1987-1988) 68.

${ }^{21}$ Huber (2011).

22 De Jong/van Soldt (1987-1988) 69-71.

23 Die Angabe zweier Monate ergibt sich dadurch, daß unbekannt ist, ob das neue Jahr vor oder nach der Herbsttagundnachtgleiche begann. In jenen Fällen, bei denen drei Monate angegeben sind, wäre der Jahresbeginn auf \pm zwei Tage mit der Herbsttagundnachtgleiche zusammen gefallen.

${ }^{24}$ De Jong/van Soldt (1987-1988) 67 datieren den Text innerhalb der letzten 75 Jahre von Ugarit, d. h. zwischen ca. 1250 und 1175 v. Chr.. Grund dafür ist, daß alle gefundenen datierbaren Texte, bei denen es sich nicht um Verträge oder Gesetzestexte handelt, in diese Zeitspanne fallen. 


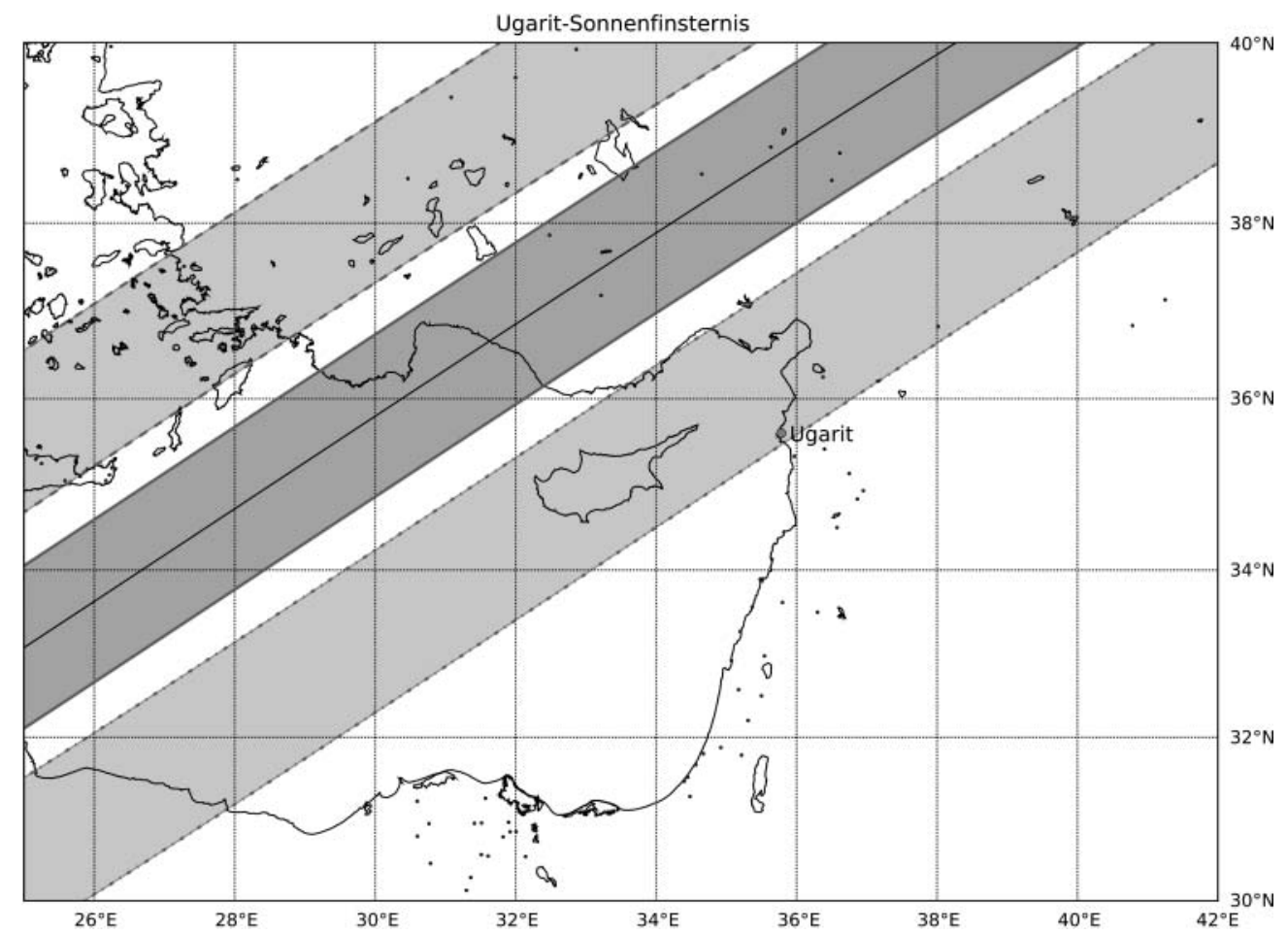

Abb. 1: Lage der Totalitätszone der totalen Sonnenfinsternis vom 5. März 1223 v. Chr. berechnet mit drei unterschiedlichen $\Delta \mathrm{T}$-Werten: mit dem unteren $\Delta \mathrm{T}$-Wert (Bereich innerhalb der gestrichelten Linien), mit dem mittleren $\Delta \mathrm{T}$-Wert (Bereich innerhalb der durchgezogenen Linien), und mit dem oberen $\Delta \mathrm{T}$-Wert (Bereich innerhalb der punkt-strichlierten Linien). Der Bereich zwischen der oberen gestrichelten und der unteren gepunkteten Kurve widerspiegelt die Unsicherheit der heutigen Berechnungen: irgendwo innerhalb dieses Bereiches verlief die Totalitätszone dieser Sonnenfinsternis. Vorausgesetzt, daß der obere $\Delta \mathrm{T}$-Wert die Gegebenheiten am besten beschreibt, wäre diese Sonnenfinsternis in Ugarit total gewesen.

Die größten Einschränkungen für eine korrekte Identifikation dieser Sonnenfinsternis bieten die aus dem Text ableitbare offensichtlich große Magnitude, daß die Finsternis im Monat Hiyyār stattgefunden hat, und die Tatsache, daß Mars über dem Horizont gestanden haben sollte. Die drei grau hinterlegten Sonnenfinsternisse sind diejenigen, bei denen alle diese Bedingungen erfüllt sind: es bleiben nur die beiden totalen Sonnenfinsternisse vom 8. Januar $1340 \mathrm{v}$. Chr. und vom 5. März 1223 v. Chr. und die ringförmige Sonnenfinsternis vom 13. März 1335 v. Chr. als mögliche Identifikationen für die Ugarit-Finsternis übrig. Da der Fundort der Tafel und die Handschrift nahe legen, daß die Tafel aus dem späten 13. bzw. frühen 12. Jh. v. Chr. stammt, muß der totalen Sonnenfinsternis vom 5. März 1223 v. Chr. der Vorzug gegeben werden. ${ }^{25}$ Unterstrichen wird das auch noch dadurch, daß nur bei dieser Sonnenfinsternis Mars in unmittelbarer Nähe der Sonne stand. Vorausgesetzt, daß der obere $\Delta \mathrm{T}$-Wert (punkt-strichlierte Kurven in Abbildung 1)

25 Die Handschrift paßt gut zu jener, die der Großteil der Dokumente aus den Priesterbibliotheken der Akropolis trägt, die in der Endzeit von Ugarit geschrieben wurden (Dietrich/Loretz [2002] 70). Auch De Jong/ van Soldt (1987-1988) 74-75 und Huber (2011) identifizieren die Ugarit-Finsternis mit der totalen Sonnenfinsternis vom 5. März 1223 v. Chr. 
die Gegebenheiten am besten beschreibt, wäre diese Sonnenfinsternis in Ugarit sogar total und Mars damit während der Totalität tatsächlich sichtbar gewesen. Die letzthin vorgeschlagene Identifikation mit der Sonnenfinsternis vom 21. Januar $1192 \mathrm{v}$. Chr. hingegen überzeugt aus astronomischer Sicht nicht: ${ }^{26}$ Mars stand tief unter dem Horizont; eine Übereinstimmung des ugaritischen Monats Hiyyār mit dem julianischen Januar hingegen wäre bei fehlerhafter Kalenderschaltung möglich.

\section{Die so genannte Ennius-Sonnenfinsternis}

Die bei Cicero überlieferte Sonnenfinsternis des Quintus Ennius wird heute meistens mit der totalen Sonnenfinsternis vom 21. Juni 400 v. Chr. identifiziert. Cicero schreibt (rep. I, $25,3)$ :

Id autem postea ne nostrum quidem Ennium fugit, qui ut scribit, anno trecentesimo quinquagesimo fere post Romam conditam Nonis Iunis soli luna obstitit et nox. Atque hac in re tanta inest ratio atque sollertia, ut ex hoc die, quem apud Ennium et in maximis annalibus consignatum videmus, superiores solis defectionis reputatae sint usque ad illam, quae Nonis Quinctilibus fuit regnante Romulo.

„Später war dies auch für unseren Ennius keine unbekannte Erscheinung. Schreibt er doch etwa 350 Jahre nach der Gründung Roms: „an den Nonen des Juni, trat der Mond vor die Sonne und [es wurde] Nacht". Und dabei zeigt sich eine so ausgeklügelte Berechnungskunst, daß ausgehend von diesem Tag, den wir bei Ennius und in den GroBen Annalen verzeichnet finden, die früheren Sonnenfinsternisse bis zu der an den Nonen des Juli unter der Regierung des Romulus eingetretenen errechnet worden sind."

Wegen zahlreicher Probleme mit dem Text ist diese Identifikation jedoch zunächst alles andere als gesichert:

1. Im Manuskript stand ursprünglich nur quinquagesimo, CCC wurde später von einer zweiten Hand dazu gefügt. Da Cicero erwähnt, daß diese Sonnenfinsternis des Ennius auch in den Annales Maximi verzeichnet war, erscheint die leicht spätere Hinzufügung der 300 Jahre allerdings eine sinnvolle Korrektur.

2. Cicero erwähnt nicht den Namen des Werkes des Ennius, aus dem er den Text zitiert. Eine plausible Annahme ist, daß die Annales des Ennius seine Quelle sind, jedoch erstaunt in diesem Zusammenhang die verwendete Bezeichnung fere, da man in Annalen eine jahrgenaue Angabe erwarten würde. Es kann jedoch sein, daß Cicero den Begriff fere deswegen gewählt hat, weil schon zu seiner Zeit unterschiedliche Bestimmungen des Nullpunktes der Zeitrechnung ab urbe condita im Umlauf waren, die um wenige Jahre differierten. ${ }^{27}$

${ }^{26}$ Dietrich/Loretz (2002) 70-72. Die Autoren bringen die auf der Tafel beschriebene Sonnenfinsternis aufgrund des ungewöhnlichen Fundortes der Tafel in den Trümmern des Palasttores mit dem Untergang von Ugarit in Verbindung.

27 Terentius Varro gibt 754/53 v. Chr. als Nullpunkt der Zeitrechnung ab urbe condita an, Dionysios von Halikarnass $752 / 51$ v. Chr. 
3. Wenn man Varro glauben darf, dann ging Ennius davon aus, daß die Gründung Roms etwa 700 Jahre vor seiner eigenen Zeit stattfand. ${ }^{28}$ Damit stellt sich die Frage, ob die Jahresangabe bei Cicero von Ennius oder von Cicero stammt und wo genau das Zitat beginnt.

Die Beschreibung der Sonnenfinsternis selbst ist wenig detailliert. Außer der Datumsangabe - die Nonen des Juni - sind keinerlei nähere Zeitangaben vorhanden. Die Erwähnung der Nacht könnte bedeuten, daß es sich um eine totale Sonnenfinsternis gehandelt hat, oder aber, daß die Finsternis gegen Abend hin stattgefunden hat. Ennius Lebensdaten werden mit 239-169v. Chr. angegeben, etwa ab seinem 20. Lebensjahr kann er literarisch tätig gewesen sein. Zieht man die unter den Punkten 2-3 angegebenen Unsicherheiten in Betracht, so bleiben die zwei folgenden großzügig gewählten Zeitfenster übrig, innerhalb deren passende Sonnenfinsternisse gesucht werden können:

1) zwischen ca. 570 und 520 v. Chr. wenn man vom Datum der Stadtgründung Roms bei Ennius ausgeht:

$$
\begin{aligned}
& 220+700=920 \quad 170+700=870 \\
& 920-870 \text { v. Chr.: Stadtgründungszeitraum nach Ennius } \\
& 920-350=570 \quad 870-350=520 \\
& 570-520 \text { v. Chr.: Datumsbereich der Sonnenfinsternis }
\end{aligned}
$$

2) zwischen ca. 420 und 380 v. Chr. wenn man vom Varronischen Stadtgründungsdatum ausgeht:

$$
\begin{aligned}
& 754-350=404 \\
& 420-380 \text { v. Chr.: Datumsbereich der Sonnenfinsternis }
\end{aligned}
$$

In beiden Intervallen lassen sich mehrere in Rom auffällige Sonnenfinsternisse finden (Tabelle 2).

\begin{tabular}{|c|c|r|r|r|r|r|r|c|}
\hline Datum & Typ & Magnitude & Beginn & Maximum & Ende & SA & SU & $\Delta$ \\
\hline 19.5 .557 & $\mathrm{~T}$ & 0.91 & $13: 18$ & $14: 41$ & $15: 56$ & $4: 50$ & $19: 13$ & +17 \\
\hline 1.11 .556 & $\mathrm{~T}$ & 0.80 & $8: 50$ & $10: 02$ & $11: 20$ & $6: 42$ & $17: 18$ & -149 \\
\hline 31.8 .534 & $\mathrm{R}$ & 0.90 & $12: 52$ & $14: 29$ & $15: 55$ & $5: 16$ & $18: 46$ & -87 \\
\hline 3.9 .404 & $\mathrm{R}$ & 0.85 & $6: 31$ & $7: 40$ & $8: 57$ & $5: 21$ & $18: 39$ & -90 \\
\hline 18.1 .402 & $\mathrm{~T}$ & 1.01 & $7: 19$ & $8: 24$ & $9: 35$ & $7: 19$ & $16: 42$ & -138 \\
\hline 21.6 .400 & $\mathrm{~T}$ & $0.89 / 0.99$ & $18: 53$ & $19: 43$ & $20: 30$ & $4: 27$ & $19: 37$ & -16 \\
\hline 14.8 .394 & $\mathrm{R}$ & 0.95 & $7: 11$ & $8: 34$ & $10: 08$ & $4: 55$ & $19: 05$ & +70 \\
\hline 12.6 .391 & $\mathrm{~T}$ & $0.81 / 0.85$ & $18: 52$ & $19: 40$ & $20: 25$ & $4: 30$ & $19: 33$ & +7 \\
\hline
\end{tabular}

Tab. 2: Datum (v. Chr.), Typ (T: total; R: ringförmig), Magnitude, Beginn, Zeitpunkt der maximalen Bedeckung und Ende der Sonnenfinsternis, Sonnenaufgangs- (SA) und Sonnenuntergangszeit (SU) gerechnet für Rom $\left(\lambda=12^{\circ} 29^{\prime} \mathrm{O}, \varphi=41^{\circ} 54^{\prime} \mathrm{N}\right)$ mit einem mittleren $\Delta \mathrm{T}$-Wert, sowie die allfällige Abweichung in Tagen des römischen Kalenders vom Sonnenstand. Die angegebenen Zeiten sind stark abhängig vom $\Delta \mathrm{T}$-Wert.

28 Varro rust. III, 1. 
Tabelle 2 faßt die wichtigsten Kenngrößen dieser Finsternisse zusammen. Die in der Tabelle dunkelgrau hinterlegten Sonnenfinsternisse sind diejenigen, bei denen die Worte „und [es wurde] Nacht“ im Quellentext am besten erklärbar wären. Die Sonnenfinsternis vom 18. Januar 402 v. Chr. war in Rom total, d. h. die hellen Planeten Venus und Merkur konnten während der vollständigen Bedeckung der Sonne durch den Mond sichtbar geworden sein. Bei der Finsternis vom 21. Juni 400 v. Chr. wurde die maximale Bedeckung der Sonne von 99\% wenige Minuten nach Sonnenuntergang erreicht, wodurch sich in Rom ein außergewöhnliches Schauspiel bot, wie schon Ginzel (1911) bemerkt hat: ${ }^{29}$ bei Sonnenuntergang war die Sonne bereits zu 89\% bedeckt, wenige Minuten nach Sonnenuntergang erfolgte ein abrupter Übergang zur Nacht, nach kurzer Zeit wurde es jedoch wieder heller und die Sterne verblaßten nochmals ehe es wirklich Nacht wurde. Auch bei der Sonnenfinsternis vom 12. Juni $391 \mathrm{v}$. Chr. erfolgte die maximale Bedeckung der Sonne erst kurz nach Sonnenuntergang, allerdings war die erreichte Magnitude hier zu gering, als daß der normale Dämmerungsverlauf wesentlich modifiziert worden wäre. Die restlichen fünf aufgelisteten Sonnenfinsternisse erzielten zwar Magnituden, bei denen man davon ausgehen kann, daß die Finsternis bemerkt wurde, aber die Bedeckung war nicht so groß, daß es tatsächlich dunkel bzw. Nacht wurde. Allerdings lassen sich bei vielen historischen Finsternisberichten Verformungstendenzen in der Überlieferung nachweisen. Eine beliebte Verformungstendenz ist die Dramatisierung des Ereignisses dadurch, daß die quantitativen Angaben gesteigert werden: partielle oder ringförmige werden $\mathrm{zu}$ totalen Finsternissen und ihre Dauer übertrieben. ${ }^{30}$ Sieht man sich die allfälligen Abweichungen des römischen Kalenders vom Sonnenstand an, so fällt auf, daß die Abweichung bei der Finsternis des Jahres 402 v. Chr. sehr groß wäre, wenn auch nicht gänzlich unmöglich. Abweichungen dieser Größenordnung sind aus den Jahren 190 und 189 v. Chr. bekannt. ${ }^{31}$ Bei den anderen beiden vorgeschlagenen Finsternissen wäre der römische Kalender in sehr guter Übereinstimmung mit dem Sonnenjahr.

Nach dieser ersten Vorselektion zeigt sich, daß die bei Cicero angegebene Anzahl Jahre seit der Stadtgründung nicht von Ennius stammen kann, sondern von Cicero. Das bedeutet, daß Ciceros Zitat des Textes von Ennius erst mit Nonis Iunis beginnt. Dieser Befund wird völlig unabhängig von dieser rein astronomischen Ableitung auch von einer metrischen Analyse der Textstelle gestützt. ${ }^{32}$ Zwischen den beiden Sonnenfinsternissen vom 18. Januar $402 \mathrm{v}$. Chr. und vom 21. Juni $400 \mathrm{v}$. Chr. die korrekte Identifikation der Ennius-Sonnenfinsternis zu wählen, ist schwierig: einzig die große nötige Abweichung des römischen Kalenders vom tatsächlichen Sonnenstand im Jahr 402 v. Chr. könnte gegen eine solche Identifikation sprechen. Die Ennius-Sonnenfinsternis fällt in einen Zeitraum, in dem in Rom ungewöhnlich viele Sonnenfinsternisse innerhalb sehr kurzer Zeit auffällig waren. Da der überlieferte Bericht keine weiteren Details enthält, stellt diese Finsternis somit einen Fall dar, bei dem eine eindeutige Identifikation nicht mit letzter Sicherheit angeboten werden kann.

29 Ginzel (1911) 213.

30 Demandt (1971) $10-18$ und 43.

31 Brind' Amour (1983) 143-147.

32 Brind' Amour (1983) 211 argumentiert, daß eine metrische Analyse des verwendeten Lateins zeige, daß es sich dabei höchstwahrscheinlich nicht um ein Zitat handle. 


\section{Zusammenfassung}

In antiken Quellen sind zahlreiche astronomische Beobachtungen überliefert, die dazu benützt werden können, absolute Daten zu gewinnen. Besonders wichtig sind in diesem Zusammenhang Sonnenfinsternisse. Hier wird ein neuer elektronisch verfügbarer Sonnenfinsterniskanon präsentiert, der speziell an die Bedürfnisse von Altertumswissenschaftlern angepasst wurde: er enthält Karten aller Sonnenfinsternisse zwischen $2500 \mathrm{v}$. Chr. und $1000 \mathrm{n}$. Chr., die im ausgewählten geographischen Bereich zwischen $\left(20^{\circ} \mathrm{N}, 5^{\circ} \mathrm{O}\right),\left(20^{\circ} \mathrm{N}\right.$, $\left.50^{\circ} \mathrm{O}\right),\left(50^{\circ} \mathrm{N}, 5^{\circ} \mathrm{O}\right)$ und $\left(50^{\circ} \mathrm{N}, 50^{\circ} \mathrm{O}\right)$ potentiell auffällig waren und somit für Identifikationen von Finsternisberichten aus diesem geographischen Bereich in Frage kommen, auch wenn sie nicht vorhergesagt wurden. Der Sonnenfinsterniskanon ist online verfügbar unter http://www.gautschy.ch/ rita/archast/solec/finsternis.html. Als Anwendungsbeispiele werden einerseits die Ugarit-Finsternis und andererseits die Ennius-Finsternis diskutiert.

\section{Summary}

Numerous astronomical records are passed down through ancient sources. Astronomical observations can be used to obtain absolute dates. Solar eclipses are the main source for such purposes. Here I present an electronic solar eclipse canon which was designed especially for the needs of historians: it contains maps of all solar eclipses in the geographical region between $\left(20^{\circ} \mathrm{N}, 5^{\circ} \mathrm{O}\right),\left(20^{\circ} \mathrm{N}, 50^{\circ} \mathrm{O}\right),\left(50^{\circ} \mathrm{N}, 5^{\circ} \mathrm{O}\right)$ and $\left(50^{\circ} \mathrm{N}, 50^{\circ} \mathrm{O}\right)$ which were potentially noticeable and therefore candidates for identifications of solar eclipse records even if the eclipse was not foretold. The solar eclipse canon is online available at http:// www.gautschy.ch/ rita/archast/solec/solec.html. As examples of use the so called Ugarit eclipse and the Ennius eclipse are discussed.

Key words: Sonnenfinsternis, Ennius, Ugarit, elektronischer Kanon

\section{Bibliographie}

Brind' Amour (1983): P. Brind' Amour, Le Calendrier Romain. Recherches chronologiques, Ottawa 1983.

De Jong/van Soldt (1987-1988): T. de Jong/W. H. van Soldt, Redating an early solar eclipse record (KTU 1.78). Implications for the Ugaritic calendar and for the secular accelerations of the earth and the moon, JVEG 30, 1987-1988, 65-77.

Demandt (1971): A. Demandt, Verformungstendenzen in der Überlieferung antiker Sonnen- und Mondfinsternisse (Abh. der Geistes- und Sozialwissensch. Kl., 1970, 7), Mainz 1971.

De Meis (2002): S. de Meis, Eclipses. An astronomical introduction for humanists (Serie Orientale Roma XCVI), Rom 2002.

Dietrich/Loretz (1976): M. Dietrich/O. Loretz, Die keilalphabetischen Texte aus Ugarit, Neukirchen-Vluyn 1976.

Dietrich/Loretz (2002): M. Dietrich/O. Loretz, Der Untergang von Ugarit am 21. Januar 1192 v. Chr.?, UF 34, 2002, 53-74.

Espenak (2004): F. Espenak, Polynominal expressions for deltaT, 2004, http://eclipse.gsfc.nasa.gov/SEhelp/ deltatpoly2004.html

Ginzel (1899): F. K. Ginzel, Spezieller Kanon der Sonnen- und Mondfinsternisse für das Ländergebiet der klassischen Altertumswissenschaften und den Zeitraum von 900 v. Chr. bis $600 \mathrm{n}$. Chr., Berlin 1899.

Ginzel (1911): F. K. Ginzel, Handbuch der mathematischen und technischen Chronologie II, Leipzig 1911.

Huber/De Meis (2004): P. J. Huber/S. de Meis, Babylonian eclipse observations from 750 BC to 1 BC, Mailand 2004. 
Huber (2006): P. J. Huber, Modeling the length of day and extrapolating the rotation of the Earth, Journal of Geodesy 80, 2006, 283-303.

Huber (2011): P. J. Huber, Dating of Akkad, Ur III and Babylon I, in: Proceedings of the 54e Rencontre Assyriologique Internationale 2008, Eisenbrauns 2011, im Druck.

Hunger/Pingree (1999): H. Hunger/D. Pingree, Astral Sciences in Mesopotamia (HdO I.44), Leiden 1999.

Neugebauer (1929): P. V. Neugebauer, Astronomische Chronologie I + II, Berlin 1929.

Neugebauer (1937): P. V. Neugebauer, Hilfstafeln zur technischen Chronologie, Kiel 1937.

Pardee (2000): D. Pardee, Les Textes Rituels II (Ras Shamra-Ougarit 12), Paris 2000.

Standish (1998): E. M. Standish, JPL Planetary and Lunar Ephemerides, DE405/LE405, Jet Propulsion Laboratory Interoffice Memorandum 312.F, 1998. 